\title{
Application of galvanotaxis of ciliated protozoan cells to automation of acute toxicity assay
}

\author{
Anton Popov ${ }^{1, *}$, Dmitriy Vinokhodov ${ }^{1}$, and Marika Rutto ${ }^{1}$ \\ ${ }^{1}$ St. Petersburg Institute of Technology (Technical University), Moskovsky pr., 26, St. Petersburg, \\ 190013, Russia
}

\begin{abstract}
One of the most negative impacts of human activity on ecosystems is extensive environmental pollution. Large territories are polluted by different chemicals, which poses threat to any agricultural activity, not to speak of unhealthy life in such places. Thus, studying and developing express bioassay methods for risk assessment of chemical substances and aqueous media are now one of the most important problems in biotechnology. These bioassay methods can be used to success in ecology, agriculture, food industry, medicine, etc. In this paper we propose a method for automation of an acute toxicity assay. This method is based on the ability of Paramecium caudatum cells to move toward the cathode under a DC electric field.
\end{abstract}

\section{Introduction}

As the test object for toxicity assessment of aqueous media we chose such protozoa as Paramecium caudatum (Ciliata). This choice is not fortuitous, because, along with their high sensitivity to heavy metals and simplicity to culture, these protozoa have long been used by biological toxicologists in the assessment of one or another property of tested media [1]. Protozoa represent a polyphyletic group of unicellular or colonial eukaryotes with a generally heterotrophic mode of nutrition. At present, due to the expanding use of methods of molecular biology and genetics, more and more evidence for allied relations between representatives of this group is being obtained and their taxonomic position is being defined more exactly. Paramecium caudatum (Ciliata) are one of the best understood classes of free-living protozoa. These organisms live in seas and freshwaters as a component of benthos and plankton, and some species live in soil and moss. Many infusoria live as commensals, symbionts, and parasites in other organisms: annelid worms, mussels, fish, amphibians, and mammals [2, 3]. Owing to their widespread occurrence in nature, fairly large size (up to $0.5 \mathrm{~mm}$ ), and specific motile behavior, ciliated paramecia have become an object of choice in many research. The diversity and differentiation of Paramecium cell structures, as well as complexity of their behavior allow paramecia to be related to a transient level of organization, specifically "a level that is still not the level of

*Corresponding author: 4qm56r@gmail.com 
multicellular differentiation but already not the level of unicellular primitivism" [4] (Fig. 1). Having a complex system of receptors and ionic channels on cell surface, as well as developed ciliature, Paramecium cells can actively react on external factors and their spatial gradients and time dynamics: temperature, density of the medium, light, radiation, electric and magnetic fields, etc. These reactions manifest themselves as taxes, kineses, and attachment reactions [5]. Taxis is the ability of microorganisms to move or orient with respect to gradient of external factors. Kinesis is an undirected change in cell motion parameters (rate and direction of motion) under the action of a factor. The following examples of tactic reaction of Paramecium caudatum can be given: geotaxis (response to gravitation field), chemotaxis (response to chemicals), rheotaxis (response to mechanical motion of the medium), and galvanotaxix (response to electric field).

It should be noted that even though these types of taxes have been known for a long time, and physiological, biochemical, and physical models have been created to explain their essence, the key issues concerning the behavioral cellular response still remain unclear $[6,2]$. Moreover, taxes are not at all limited to the abovementioned protozoan types: the higher the level of organization, the more challenging is to decompose into elementary components and model the behavioral response of a biological object. Different Paramecium species much differ in sensitivity to one or another external factor; of importance are also conditions of culturing, age of the culture (physiological state, clonal cycle), and adaptation to the studied factor. Due to the existence of taxes and kineses, studying the motile activity of cells can in itself provide a lot of information on the physiological state of cells in given conditions, and, consequently, allow one to assess the intensity of stimuli. Thus, research on taxes is not only of theoretical, but also of applied interest.

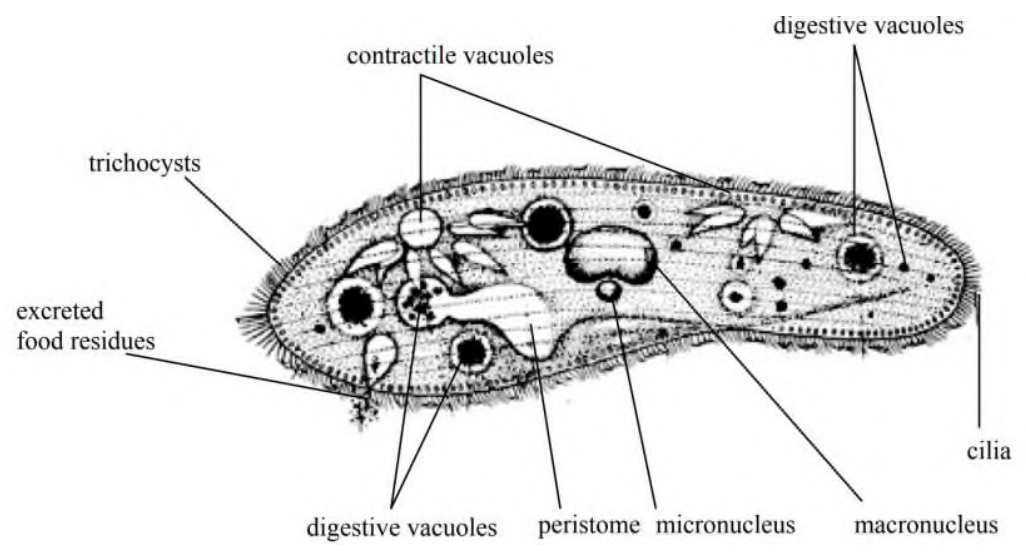

Fig. 1. Photograph and schematic structure of a typical Paramecium cell [2].

\section{Medium and chemicals used}

The Paramecium caudatum culture, clone 94A B6-18, was kindly provided by the Chair of Engineering Protection of the Environment, St. Petersburg State Electrotechnical University. Culturing was performed in a hay broth.

The culture medium was divided into $100-\mathrm{mL}$ portions that were placed into $250-\mathrm{mL}$ conical flasks and left to stand for a day to let hay bacteria which are nutrients for growing ciliates to develop. The Paramecium caudatum culture $(5 \mathrm{~mL}$, cell concentration 1000 cells $/ \mathrm{mL}$ ) was then added to the flasks, after which they were covered with cotton woolgauze plugs. The culture was stored at room temperature, its washing and concentration 
were performed using a previously designed device and a traditional washing procedure (in volumetric flasks) $[6,7]$. No culture synchronization was applied before toxicological experiments. Heavy metal salts (CdI2, $\mathrm{CuSO} 4, \mathrm{~Pb}(\mathrm{NO} 3) 2, \mathrm{CrCl} 3$, and $\mathrm{NiCl} 2)$ were used as model toxins.

\section{Device for automated toxicity assay of environmental samples (TOVS)}

The progress in materials science, microelectronics, and applied software engineering has made possible design of convenient and fairly inexpensive devices for automated acquisition, processing, and storage of large bodies of information. For adequate responses toxicological experiments should involve multiple parallel tests, and, therefore, automation of some experimental stages can strongly facilitate research work. At present devices that allow fully automated bioassays have been developed. Ideally, such devices should allow one to (i) maintain necessary conditions throughout the entire experiment; (ii) control responses of biological objects to stimuli (test reaction reading), (iii) and record the strength of responses (test criteria) in dimensional or dimensionless values, and (iv) apply software algorithms for statistical processing of resulting data. The device (TOVS) designed at our chair makes it possible to control the motion of Paramecium caudatum cells placed in a special glass cuvette. The motion and density of the cell cloud in the cuvette are detected, as will be shown below, by turbidimetry (by the degree of front diffuse light scattering by cells, measured with a photoelectric cell). The device is portable (Fig. 2). It has a liquid crystalline display and controllers connected to the photoelectric cell. The current direction in the cuvette is controlled with a microcontroller, and tuning of the device is performed from a computer via a COM port. The direction of motion of Paramecium caudatum cells is controlled by varying the polarity of the graphite electrodes installed in the cuvette. Readings of the photoelectric cell set up opposite to the light source are taken continuously. The electrode polarities are changed at preset time intervals (from $30 \mathrm{~s}$ to $5 \mathrm{~min}$ ). The concentrated Paramecium cell cloud crosses the light beam axis, forming the photoelectric signal of a certain intensity. The photolelectric signals are acquired by a computer and tabulated in Excel tables for further processing.

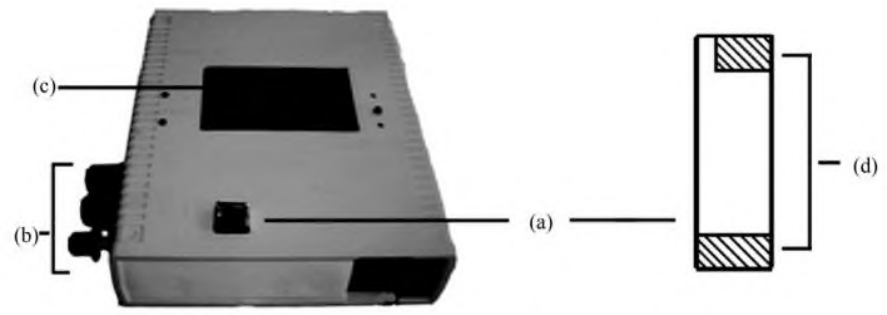

Fig. 2. Device for integral toxicity assessment TOVS [6]: (a) optical cuvette with graphite electrodes; (b) zero-response controllers; (c) display; and (d) graphite electrodes.

\section{Device for automated toxicity assay of environmental samples (TOVS)}

First we studied the response of the device in the absence of toxic compounds. The varied parameters were cell concentration, culture age, cell washing procedure, and mineral composition of the medium. This work was performed with the aim to find a reproducible and readily interpretable response of the device to a nontoxic control medium. As expected, 
changes in cell concentration in the sample had the strongest effect on the response. On the one hand, the lower the concentration of cells in the cuvette, the weaker is light scattering, and, therefore, the "efficient" signal of the device becomes more difficult to distinguish. On the other hand, at high concentrations (about $4000 \mathrm{cells} / \mathrm{mL}$ ), strong hydrodynamic fluxes of the medium develop because of the motion of a great number of objects and such fluxes disintegrate the cloud and induce random cell motion. Probably, the nature of these hydrodynamic fluxes, too, will be strongly dependent of the geometry of the cuvette. The optimal working concentration range was 1000-2000 cells $/ \mathrm{mL}$, and the data presented in what follows all relate to this range. It should also be mentioned that at concentrations higher than 2000- 5000 cells $/ \mathrm{mL}$ cells become to be much sensitive to certain types of toxins, which is sometimes manifested in a complete absence of toxic effect. This is especially evident at low concentrations of toxins; therewith, in experiments with single cells, toxic effects developed fairly rapidly (immobilization, deformation of certain structures, death, lysis), whereas in experiments with cell suspensions, toxins at the same concentrations uniformly distributed between organisms and did not reveal any detectable effects. To assess the effect of the age of the culture on the behavior of cells in the cuvette, we studied 2-, 5-, 10-, and 14-day Paramecium cultures to find that the age of the culture. However, it should be taken into account that the age and culturing conditions may essentially affect the sensitivity of cells to certain classes of toxins. A correlation between sensitivity and age is well documented, especially for biological objects whose physiological parameters tend to considerable vary with time (cell aging in higher animals, clonal cycles in protozoans, etc.). Washing of cells from nutritive media is always recommended, because suspensions and any organics can react with substances to be tested, thereby attenuating their toxic effect and affecting the bioassay results. All toxicological experiments were first performed by a classical procedure. Each flask was examined under a microscope, dead cells at each time interval were counted visually, and these data were used to construct plots of cell death dynamics and estimate such toxicological parameters as lethal exposures, such as LT (lethal exposure time) and LC (lethal exposure concentration). It was shown that all the standard mineral media used for culturing and cell washing are suitable for experiments and have no effect on the sensitivity of the organisms. It should be noted here that, considering cell physiology, for cells to show normal motile activity and ability for galvanotaxis, of primary importance are the concentrations of potassium and calcium ions in the medium. Exchange of these ions through protein channels in cell membranes plays, along with other regulatory and sensory functions, the key role in maintaining cell motility [6]. Further on in all experiments we used the standard LozinLozinskii mineral medium for cell culturing and washing: To prepare $1 \mathrm{~L}$ of the medium we took $0.1 \mathrm{~g}$ of $\mathrm{NaCl}, 0.01 \mathrm{~g}$ of $\mathrm{KCl}, 0.01 \mathrm{~g}$ of $\mathrm{CaCl} 2,0.01 \mathrm{~g}$ of $\mathrm{MgCl}$, and $0.02 \mathrm{~g}$ of $\mathrm{NaHCO} 3$.

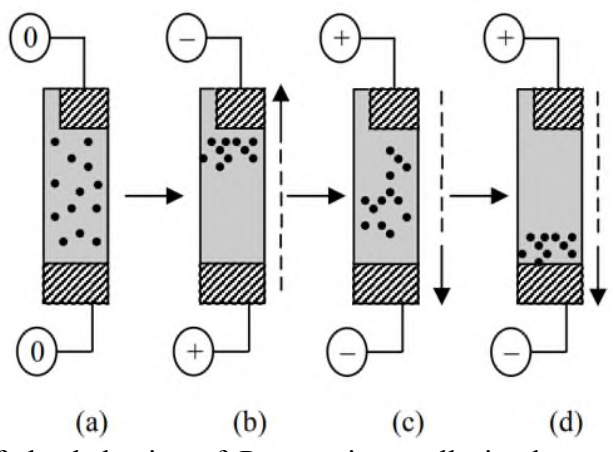

Fig. 3. Schematics of the behavior of Paramecium cells in the working cuvette under multiple electrode polarity reversal (dashed arrows show cell motion directions, sings - charges of electrodes). 


\section{Behavior of paramecium cells in the working cuvette of the device. TOVS response}

The working cuvette of the device is made of glass. The geometric dimensions of the cuvette are $45 \times 10 \times 10 \mathrm{~mm}^{3}$. Graphite electrodes are mounted in the top and bottom. The working cuvette is filled with a suspension of washed cells and placed into the sample chamber of the device. In the first case, the electrodes are not energized; in the second case, the electrodes are energized, and paramecia concentrate at the top electrode, namely cathode (Fig. 3b). Further the polarity of the electrodes is reversed, and cells move to the bottom electrode (Fig. 3c) and concentrate there (Fig. 3d). In what follows these motions of the cloud of concentrated cells from one electrode to the other are called "runs".

During testing such runs (Figs. 3b-3d) are repeated many times. The software controlling the device allows on to set the total number of runs and the time of one run, when the field direction in the cuvette is not changed. Our experiments showed that too long runs (longer than $1 \mathrm{~min}$ ) are unadvisable, because in this case the cells concentrated at the top electrode start to settle down. The random peaks associated with this process give no insight into the toxicity of the medium. If runs are too short (shorter than $30 \mathrm{~s}$ ), paramecia have no time to concentrate near the cathode. The optimal time, when all cells can concentrate at the cathode is $60 \mathrm{~s}$. It was shown that the rate of cell motion is primarily dependent on the parameters of the medium (viscosity, density), as well as on the electric field strength $(\mathrm{V} / \mathrm{cm})[6-8]$. Note also that the sensitivity of cells to toxins depends on the time of exposure to direct current, and, therefore, varying this time can be used as an additional physical load in bioassay experiments. Not infrequently, the higher the field strength of (or longer the exposure time to) the electromagnetic field in the medium, the more sensitive is the test object in hands to the toxin being tested, and the more rapidly the test reaction reveals itself. Sensitivity enhancement by exposure of the test object to nearextreme conditions (depending on the adaptation conditions of the test object) is well known in the literature.

These extreme factors may include critical temperatures, chemical composition of the medium, illumination, electromagnetic field strength, etc. Exposure to an extreme factor can much enhance (or, sometimes, attenuate) the sensitivity of the test object to a negative impact of this factor. The response of the device is a signal coming from the photoelectric cell every 0.25 seconds. The signal measures the optical density of the medium, which correlates with the number of cells crossing the light source- photoelectric cell axis; the dependence of response on time is plotted on the display in real time. Thus, as the device response is directly related to the density of the cell cloud, a higher peak corresponds to each even run, when the cathode is on top. Odd runs are associated with fairly low, random peaks, because the cell cloud on its downward motion has a more diffuse front (Fig. 4).
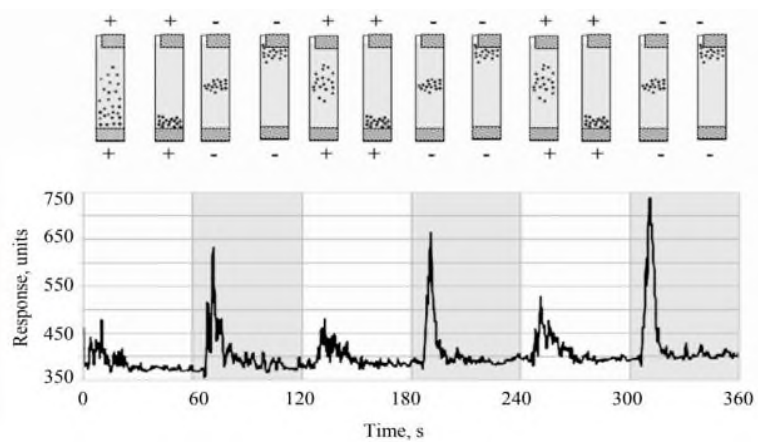

Fig. 4. Plot of the TOVS responses on a nontoxic medium against time. Cell concentration ca. 2500 cells $/ \mathrm{mL}$. The cell positions in the cuvette and the response plot are coincident. 
Thus, for toxicity assessment we used the dynamics of change in the relative height of peaks associated with the upward motion of a dense cell cloud to the top electrode. These peaks are regular and well defined. It was shown that they appear every 73 seconds after the starting point. The area and amplitude of these peaks depend on the character of motion, ae well as density and motion rate of the Paramecium cell cloud. Experiments for choosing optimal characteristics of the cell culture (cell concentration, culture age) were performed with the aim to ensure the best conditions for obtaining stable peaks on even runs. Cell cultures 2-5 days of age were used. Tests with varied cell concentrations were set up on each age culture. The optimal cell concentration was found to be about 1500 cells $/ \mathrm{mL}$. As seen from Fig. 5, from the change in the height of even peaks (an envelope peak) we already can draw conclusions as to whether the tested solution is toxic to paramecia. And, taking into account that unicellular organisms may be several orders of magnitude more sensitive to the same chemical compounds than multicellular [2], this primary qualitative estimate is close to established standards for the maximum allowable concentrations of certain compounds and their mixtures. Comparison of the response plots from different experiments can be performed, say, by directly comparing the dynamics of the change in relative peak heights (more specifically, envelope curve patterns), and this is what we will do in the next section. Thus, for the characteristic parameter to be compared in our experiments we chose the time when the relative peak height falls to $50 \%$ of its initial height. This test criterion was chosen by analogy with such traditional toxicological parameters as lethal exposures (LT50 and LT100).
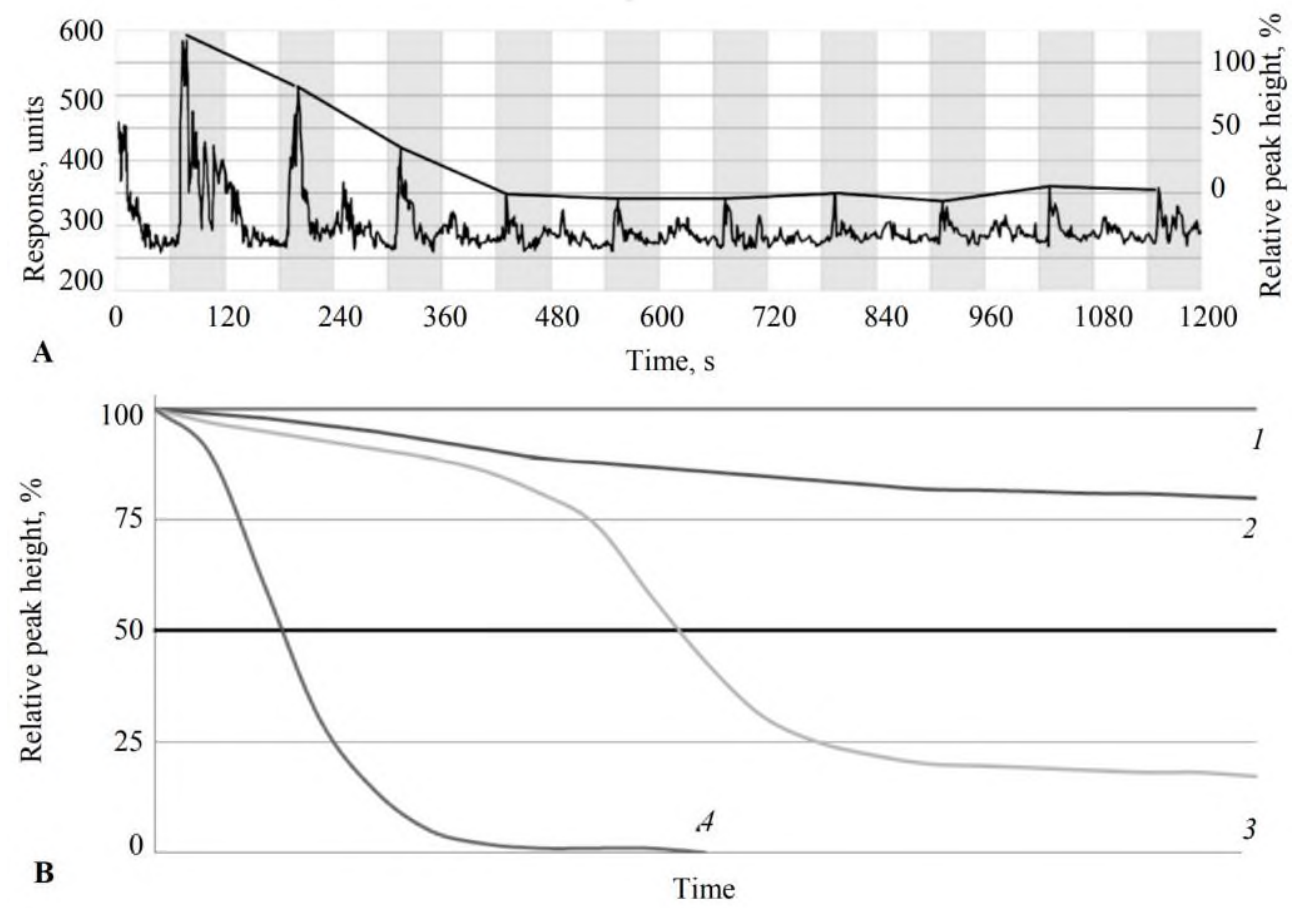

Fig. 5. (A) Testing the sample containing $0.005 \mathrm{~g} / \mathrm{L}$ of $\mathrm{CdI}_{2}$ and drawing an envelope response curve. In what follows, we use only envelope response curves. (B) Theoretically possible envelope TOVS response curves. 


\section{Conclusions}

Thus, the device we designed allows automated control of the behavior of Paramecium caudatum cells in toxic media and can provide a perspective tool for express assessment of the integral toxicity of various environmental samples. In principle, the developed method can be adapted to any organisms with a stable galvanotactic reaction. However, in view of the fact that statistically reliable results can only be obtained for samples that exhibit acute toxicity (toxicity causing, sooner or later, death of test objects), the method does not work at concentrations causing chronic toxicity but not lethal. For this purpose, other bioassays are required.

\section{References}

1. D.O. Vinokhodov, Doctoral (Biol.) Dissertation (St. Petersburg, 2001)

2. K. Hausmann, Protozoologie (New York: Thieme, 1985)

3. A.V. Ivanov, Yu.I. Polyanskii, A.A. Strelkov, Big Manual on Invertebrate Zoology (Moscow: Vysshaya shkola, 1981)

4. L.N. Seravin, Protozoa, What Is It? (Leningrad: Nauka, 1984)

5. H.H. Dale, J. Physiol. 26, 5, 291-361 (1901)

6. A.V. Popov, D.O. Vinokhodov, A.A. Seregina, High Technologies, Research, Industry, St. Petersburg: Politekh. Univ. 2, 14-17 (2010)

7. M.V. Vinokhodova, A.V. Popov, A.A. Seregina, P.A. Zubanov, D.O. Vinokhodov, Izv. Sankt. Peterb. Tekh. Univ. 13, 39, 61-63 (2012)

8. I.S. Zakharov, A.V. Pozharov, T.V. Gurskaya, A.D. Finogenov, Biosensor Systems in Medicine and Ecology (St. Petersburg: Sankt. Peterb. Gos. Univ. Telekom., 2003)

9. N. Ogawa, H. Okua, K. Hashimoto, M. Ishikawa, J. Theor. Biol. 242, 314-328 (2006)

10. K.A. Robinson, J. Cell. Biol. 101, 6, 2023-2027 (1985)

11. L.A. Zaliznyak, V.E. Kokova, Tsitologiya 34, 4, 62 (1992)

12. K.A. Samoilova, Dokl. Akad. Nauk SSSR 155, 3, 670-672 (1964)

13. V. Ageev, I. Doltornyazov, Ptitsevodstvo 8, 41-42 (1988)

14. A.G. Kazantseva, Cand. Sci. (Tech.) Dissertation (St. Petersburg, 2010) 